\title{
Kernos
}

Revue internationale et pluridisciplinaire de religion grecque antique

9 | 1996

Varia

\section{Gérard CAPDEVILLE, Volcanus. Recherches comparatistes sur les origines du culte de Vulcain}

\author{
Vinciane Pirenne-Delforge
}

\section{OpenEdition \\ Journals}

\section{Édition électronique}

URL : http://journals.openedition.org/kernos/1195

DOI : 10.4000/kernos. 1195

ISSN : 2034-7871

\section{Éditeur}

Centre international d'étude de la religion grecque antique

Édition imprimée

Date de publication : 1 janvier 1996

ISSN : 0776-3824

\section{Référence électronique}

Vinciane Pirenne-Delforge, « Gérard CAPDEVILLE, Volcanus. Recherches comparatistes sur les origines du culte de Vulcain », Kernos [En ligne], 9 | 1996, mis en ligne le 21 avril 2011, consulté le 24 septembre 2020. URL : http://journals.openedition.org/kernos/1195; DOI : https://doi.org/10.4000/kernos.1195 
184 pour les femmes de la même période. Il y a là des indications non négligeables quand on entend évaluer le rôle que les idées religieuses jouaient dans la Cité.

Jules LABARBE

(Université de Liège)

Gérard CAPDEvILLE, Volcanus. Recherches comparatistes sur les origines du culte de Vulcain, École française de Rome, Palais Farnèse, 1995. 1 vol. 16 x 24, 5 cm, 519 p., 29 fig., 2 cartes (BEFAR, fasc. 288). ISBN : 2-72830272-3.

Le point de départ de ce livre très érudit est le constat que l'enseignement classique relatif à Vulcain cadre mal avec les renseignements fournis par la pratique religieuse. La méthode pour poser un tel problème est résolument comparatiste. Le lecteur voyage dès lors de Rome en Crète, à Chypre, en Grèce, pour en terminer par l'Étrurie et un bref retour à Rome. Après une introduction très courte, l'ouvrage s'articule en trois grandes parties; il se referme sur une conclusion substantielle, une bibliographie très abondante, répartie en fonction des subdivisions de l'exposé, les 29 illustrations qui soutiennent le propos, une carte de Chypre et une de la Crète, et quatre riches index (locorum, geographicus, nominum deorum et bominum, rerum notabilium), très utiles pour s'orienter dans ce livre foisonnant.

La première partie, intitulée Filii volcani, étudie quatre légendes (Servius Tullius, Caeculus, Romulus et Cacus) dont l'analyse met au jour une même structure : un héros est engendré par Vulcain ou par un substitut igné; élevé dans un milieu de bergers, il prend la tête d'un groupe de jeunes gens pour vivre de rapines aux marges des centres urbains, avant d'aller fonder une cité dont il prend le pouvoir et où le dieu reste important. Un tel schéma pourrait avoir une origine étrusque, mais c'est en élargissant le propos que l'A. entreprend de parcourir le chemin, difficile et souvent hasardeux, qui mènerait aux origines d'un dieu.

Les Dactyles, présents sous une forme latinisée autour de Caeculus, nous conduisent en Crète, leur terre de prédilection (surtout le Mont Ida), à la poursuite du mystérieux $F \varepsilon \lambda \chi \alpha ́ v o \varsigma$ qui sert de titre à la deuxième partie du livre. Le nom de Velchanos est proche de celui de Volcanus, et les quelques témoignages - peu parlants, il est vrai - concernant ce dieu s'étendent du VIIe ou VIe s. av. J.-C. au IIe ou IIIe $s$, de notre ère. Ils concernent quatre cités importantes de la Crète centrale. Comme Velchanos est inconnu ailleurs dans le monde grec, l'A. en déduit qu'il doit être d'origine préhellénique et que le petit temple hellénistique d'Haghia Triada, qui lui est consacré, est voué à un culte qui remonte à l'époque minoenne. Pour ces temps anciens, le dieu - que des monnaies de Phaistos de 322-300 av. J.-C. montrent assis dans la fourche d'un arbre - est interprété comme une divinité de la végétation présidant au renouveau printanier. L'arbre, exprimant la puissance reproductrice de la Terre-Mère, incarnerait "le principe féminin " symboliquement fécondé par l'éclair, figuré par la double hache, "principe mâle » (p. 170). Ce partenaire masculin de la Grande Déesse aurait ensuite revêtu la forme du taureau, comme en attestent les légendes de Pasiphaé ou d'Europe. Enfin, c'est la forme humaine qui aurait prévalu, pour illustrer une hiérogamie où l'arbre est toujours bien présent, symbole de l'union du ciel et de la terre. Ainsi, l'arbre qui accueille Velchanos sur les monnaies 
serait le lieu de l'union du dieu et de la déesse, qui pourrait s'être appelée Hellotis. Avec l'arrivée des Doriens, ce couple à dominance féminine serait devenu le couple

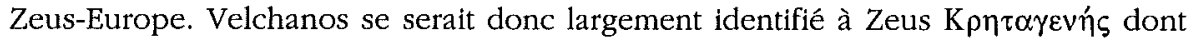
le mythe crétois originel pourrait se trouver dans le mythe de succession de la Théogonie hésiodique. Le parcours initiatique de ce Zeus ressemble à celui des premiers rois du Latium, fils de Volcanus, mais aussi aux pratiques initiatiques attestées pour les jeunes Crétois de l'histoire. Quant à affirmer qu'à haute époque, c'est précisément Velchanos qui patronnait ce processus, il y a un pas que l'A. franchit avec témérité (p. 214). En fait, si le schéma initiatique est similaire - et largement répandu en dehors du Latium et de la Crète ! -, l'implication d'un Volcanus/Velchanos dans l'initiation crétoise est une hypothèse certes séduisante, mais fragile. De même, les théories évolutives de la «théologie » crétoise primitive reprises par l'A. sont hautement spéculatives et relèvent encore des interprétations optimistes et «volontaristes » des premiers fouilleurs de Cnossos.

On peut s'étonner, vu l'abondante bibliographie que propose l'A., de ne voir aucune mention des importantes contributions de Nanno MARINATOS sur la religion crétoise. De même, on aurait aimé avoir son avis sur l'interprétation des plaques en métal martelé retrouvées en grand nombre sur le site de Kato Syme (à quelque $70 \mathrm{~km}$ à l'est de Phaistos à vol d'oiseau) et interprétées par Angeliki LEBESSI, l'archéologue qui a fouillé le sanctuaire, comme des ex-voto offerts à la suite de

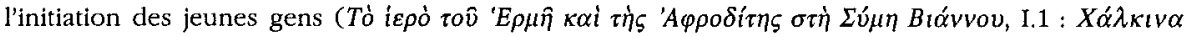

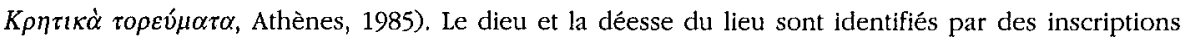
hellénistiques comme étant Hermès et Aphrodite.

La découverte, dans la grotte de l'Ida, d'un petit bronze géométrique représentant une scène d'« enlèvement » à proximité d'un navire, de même que le décor similaire d'un anneau d'or trouvé près d'Héracleion conduisent l'A. à proposer une nouvelle étude de la légende de Thésée. L'analyse minutieuse de l'imposant dossier documentaire permettrait de reconnaître le schéma type du mythe du premier roi, à l'état de trame pour bon nombre de traits. C'est jusqu'à Délos que les qualités d'un hypothétique Velchanos auraient été assumées par Apollon.

Était-il vraiment nécessaire de reprendre systématiquement l'analyse du dossier «initiatique » de Thésée après l'ouvrage de Cl. CALAME, Thésée et l'imaginaire athénien (Lausanne, 1990), que l'A. ne cite pas? Il est clair que la perspective des deux travaux est fondamentalement différente, mais n'est-il pas sain de confronter des orientations méthodologiques diverses quand, de surcroît, elles présentent certaines lignes de convergence? De plus, la volonté de reconnaître dans le cycle de Thésée le schéma mythique au centre de la thèse conduit à certaines lectures contestables. Le personnage d'Ariane ne joue pas le rôle de la princesse qui confère la souveraineté. Par contre, c'est un personnage fortement sexualisé, ce qui correspond bien à un cadre initiatique prématrimonial. La couronne, qu'Ariane donne à Thésée et dont la fabrication est parfois attribuée à Héphaïstos (voilà le Volcanus grec !), pouvait être un don de Dionysos, un dieu dont l'apparition dans ce mythe pose bien des problèmes et que l'A. ne nomme jamais.

C'est à Chypre que nous conduit ensuite la piste de Velchanos. Une inscription

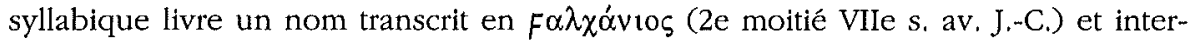
prété par l'A. comme un nom de mois. Un relief en terre cuite présente en outre un thème iconographique proche de celui des monnaies de Phaistos. Vu les contacts attestés entre l'île et sa voisine crétoise, la religion de Chypre est passée au crible afin d'y déceler des pratiques initiatiques analogues. Un obscur rituel d'Amathonte, dont les protagonistes reçoivent les noms de Thésée et d'Ariane, en conserverait la trace, 
de même que des banquets (?). Le parèdre de la Grande Déesse de l'île, dont l'activité métallurgique conviendrait bien à un Volcanus indigène, reproduirait le "schéma" du Velchanos crétois, peu à peu identifié à Apollon. Héphaïstos est ensuite appelé à la barre, en ses cultes lemnien et athénien, où l'A. retrouve, une fois encore, avec des déformations qui seraient dues à autant de réinterprétations, les données mises au jour dans les îles égéennes.

La troisième et dernière partie nous ramène en Étrurie, à la recherche d'une définition du dieu*Velchans, en un long développement dont je ne suis pas à même d'évaluer la pertinence, mais qui fait appel à la fois aux légendes et aux cultes locaux. Et c'est avec la conclusion que l'A. reprend l'ensemble des acquis pour redéfinir la personnalité du Volcanus latin.

Ce travail, dont la présentation est irréprochable, est une véritable thèse qui met à son service un comparatisme fouillé et une remarquable documentation. Une foule de questions, dont l'importance et l'intérêt sont variables, sont aussi abordées dans les notes. La plupart des textes anciens discutés - dont l'importance pour le propos est également très variable - sont reproduits intégralement dans leur version originale avec, systématiquement, une traduction de l'A. en note. Une telle recherche est très séduisante et satisfait l'aspiration à comprendre des traditions éclatées dans une mise en perspective globalisante. L'A. propose maints rapprochements dont la convergence emporte souvent l'adhésion. Néanmoins, malgré la prudence dont il fait preuve en rappelant fréquemment le caractère hypothétique de bon nombre d'interprétations, on est pris de vertige devant une recontruction dont les assimilations comparatistes ne permettent pas de justifier toutes les étapes.

Vinciane PIRENNE-DELFORGE (Université de Liège)

Augusto Fraschetri, Rome et le prince. Traduction française par Vincent Jolivet, Paris, Belin, 1994. 1 vol. 14 x 20,5 cm, 380 p. (L'Antiquité au présent). ISBN : 2-7011-1478-0.

Nicole Loraux et Yan Thomas ont eu l'excellente idée d'accueillir dans leur collection, L'Antiquité au présent, une version française de l'ouvrage d'Augusto Fraschetti, Roma e il principe (Roma-Bari, 1990). Le sujet n'est pas neuf : étudier de quelle manière et dans quelle mesure la vie du citoyen romain a été modifiée, spécialement dans le domaine religieux, par les changements dus au passage de la République au Principat, étape cruciale de l'histoire romaine que R. Syme a naguère qualifiée à juste titre de Révolution romaine. C'est le problème le plus épineux posé par le Principat, qui a fait couler des flots d'encre, qu'affronte l'A. : le rapport du Princeps et de l'Urbs et la manière dont s'insèrent dans les structures existantes de l'État romain éléments politiques et religieux pour former l'auctoritas Augusta. La nouveauté propre à cette étude, c'est l'angle selon lequel la matière est abordée. L'A. n'hésite pas à remettre en débat des questions qui passent généralement pour résolues depuis longtemps - en fait depuis Mommsen - et à faire un sort à des problèmes généralement considérés comme de simples curiosités d'érudits ou d'antiquaires. Fondée une quantité de textes minutieusement étudiés, sa réflexion 